\title{
Sex differences in dietary consumption and its association with frailty among middle- aged and older Australians: a 10-year longitudinal survey
}

Xiaoyue $\mathrm{Xu}^{1,2,3^{*}}$, Sally C Inglis ${ }^{3}$ and Deborah Parker $^{3}$

\begin{abstract}
Background: Nutritional status has been considered as a key factor in preventing the development of the frailty syndrome. However, sex-specific dietary consumption transition over time and how it impacts of frailty status are unclear.

Method: We assessed 113,039 adults (aged 50 years and over) from the 45 and Up Study who had completed both baseline (2006-2009) and follow-up (2012-2015) surveys. Dietary consumption was assessed by a short food frequency questionnaire. Frailty was identified by the FRAIL scale. Multinomial regression models were used to examine the association between a long-term dietary consumption and frailty, stratified by sex.

Results: Of a total of 113,039 participants, females had a higher percentage of pre-frailty and frailty than males (prefrailty: $35.5 \%$ for female and 30.1\% for male; frailty: $4.86 \%$ for female and 3.56\% for male). As age increased, males had significant decreases in overall dietary risk scores, while females had significant increases in overall dietary risk scores. Males and females with a long-term consumption of adequate fruits, high grains or had a variety of foods were related to a low risk of frailty. Females with a long-term consumption of adequate vegetables or high lean meats and poultry were related to a low risk of frailty. Females with an unhealthy diet at both surveys [Relative Risk Ratio $(\mathrm{RRR})=1.32,95 \% \mathrm{Cl}: 1.18 ; 1.49$ ], and those with unhealthy diet at either surveys (RRR $=1.28,95 \% \mathrm{Cl}: 1.12 ; 1.47$, $\mathrm{RRR}=1.19,95 \% \mathrm{Cl}: 1.04 ; 1.37)$ had a higher risk of frailty compared to those had a long-term healthy diet. No association were found between overall dietary risk and frailty for males.

Conclusion: Males and females changed their dietary consumption as they age. These changes affect its association with frailty, particularly for females. Sex-specific dietary advice in prevention of frailty needs to be further developed.
\end{abstract}

\footnotetext{
* Correspondence: luna.xu@unsw.edu.au

${ }^{1}$ School of Population Health, University of New South Wales, Sydney, New South Wales, Australia

${ }^{2}$ The George Institute for Global Health, Sydney, New South Wales, Australia

Full list of author information is available at the end of the article
}

C C The Author(s). 2021 Open Access This article is licensed under a Creative Commons Attribution 4.0 International License, which permits use, sharing, adaptation, distribution and reproduction in any medium or format, as long as you give appropriate credit to the original author(s) and the source, provide a link to the Creative Commons licence, and indicate if changes were made. The images or other third party material in this article are included in the article's Creative Commons licence, unless indicated otherwise in a credit line to the material. If material is not included in the article's Creative Commons licence and your intended use is not permitted by statutory regulation or exceeds the permitted use, you will need to obtain permission directly from the copyright holder. To view a copy of this licence, visit http://creativecommons.org/licenses/by/4.0/ The Creative Commons Public Domain Dedication waiver (http://creativecommons.org/publicdomain/zero/1.0/) applies to the data made available in this article, unless otherwise stated in a credit line to the data. 


\section{Background}

The prevalence of frailty is higher in older adults but is not considered as a part of normal ageing. Frailty is a medical condition characterised by a functional (physical and cognitive) decline, that requires the need for assistance to perform the daily living activities [1]. There is no curative treatment for frailty, so the efforts have focused on the prevention and palliation of symptoms, with focus on effective physical and nutritional interventions or control of polypharmacy [2].

The factors responsible for the development of frailty is still a matter of intense debate, but there have been several factors noted, such as sarcopenia or muscle mass loss. In this line, nutritional status has been identified as a key factor in preventing the development of frailty syndrome. Previous studies predominantly cross-sectional in design, have provided evidence on the relationship of micronutrients (e.g. vitamins $\mathrm{D}, \mathrm{E}$ and $\mathrm{C}$, and folate), macronutrient (e.g. protein), dietary pattern (e.g. Mediterranean diet), dietary quality and frailty [3]. Overall, a diet with low energy intake, insufficient consumption of protein, micronutrients such as vitamin D, C, calcium and omega-3 fatty acids is associated with an increased risk of frailty development [4]. Control or balance in nutritional status is essential to prevent sarcopenia and further frailty development [3, 4]. The relationship between nutrition and frailty is likely bidirectional that poor nutrition or malnutrition might contribute to frailty, or conversely, frailty may contribute to poor nutrition or malnutrition [5-7].

As people age, they may eat less and make difference food choices. It is unclear whether the dietary consumption transition over the life course may impact on the frailty status of older individuals. In addition, studies have indicated sex/gender differences in food choice, and in energy and nutrients intake [8]. It is also possible that dietary consumption may differ by sex as people age, which may have different impacts on frailty status. Although it has been clearly stated in the previous research that sex-specific analyses of research data should be the norm [9], most studies failed to report this, both in Australia and internationally.

To better understand the sex-specific frailty status among middle-aged and the older Australian population, also to understand the dietary consumption transition and how it impacts of frailty status, this study used data from the longitudinal 45 and Up Study to 1) evaluate sex-specific frailty status, 2) track changes of dietary consumption by frailty status and sex, and 3) examine the association between long-term dietary consumption and frailty, stratified by sex.

\section{Method}

\section{Study population}

The Sax Institute 45 and Up Study baseline and followup data were used. The 45 and Up Study is the largest ongoing study of healthy ageing ever undertaken in the Southern Hemisphere and is designed to understand how Australians are ageing. Participants were randomly sampled from the Department of Human Services enrolment database [10]. A total of 267,153 men and women aged 45 and over across New South Wales, Australia were recruited and surveyed in 2006-2009, representing about $10 \%$ of this age group. Upon recruitment, participants provided consent for future follow-up. The first follow-up survey data were collected between 2012 and 2015. At both time points, socioeconomic, health behaviour and health related information were collected via a comprehensive questionnaire that was mailed to people. Details of the 45 and Up Study sampling process are described elsewhere [11]. The baseline and follow-up questionnaires are available at Sax Institute website (https://www.saxinstitute.org.au/our-work/45-up-study/ questionnaires/). In the present study, we included those participants who completed both baseline and follow-up questionnaires on dietary consumption. A total of 113, 039 participants were included in the analysis.

\section{Outcome variable: frailty}

We used the FRAIL scale to identify frailty. The FRAIL scale was developed by the Geriatric Advisory Panel of the International Academy of Nutrition and Aging, and can be administered by conducting a brief interview or constructed from self-reported survey data [12]. Previous studies have shown that the FRAIL scale score is predictive of mortality and disability [12], and the scale has been tested as a valid and responsive tool to identify frailty with suitability for use in longitudinal studies of older Australians [13].

The FRAIL scale is based on deficits in five domains: Fatigue; Resistance (ability to climb one flight of stairs); Ambulation (ability to walk one block), Illnesses (greater than 5) and Loss of Weight (>5\%). Participants were scored positive as 1 for each of these five domains, so the FRAIL ranges from 0 (not frail) to 5 (most frail) [13, 14]. The FRAIL scale was used to categorize participants as healthy (score 0 ), pre-frail (score of 1-2) or frail (score of 3-5).

These five domains are matched with the 45 and Up study questionnaire. Specifically, for 'Fatigue': participants scored positive if they responded, "most of the time" or "all of the time" to the questionnaire "during the past 4 weeks, about how often did you feel tired out for no good reason?" For 'Resistance': participants scored positive if they responded "limited a little" or "limited a lot" on their ability to climb one flight of stairs. For 
'Ambulation': participants scored positive if they responded "limited a little" or "limited a lot" on their ability to walk $100 \mathrm{~m}$. For 'Illness': if participants reported more than five of the following diseases: Alzheimer's diseases or dementia, angina pectoris or heart attack, depression, arthritis (including osteoarthritis and rheumatoid arthritis), asthma, bronchitis or emphysema, diabetes, hypertension, osteoporosis and stroke. For 'Loss of weight': participants scored positive if their selfreported weight decreased by $5 \%$ or more between baseline and follow-up survey. The domains of 'Fatigue', 'Resistance', 'Ambulation' and 'Illnesses' were identified from follow-up data. 'Loss of weight' was identified from both baseline and follow-up data. The frailty status was identified at follow-up data based on all of five domains.

\section{Predictive variable: a long-term dietary consumption}

Dietary consumption was identified from seven food components based on the Australian Dietary Guideline (ADG): vegetable, fruit, grains, lean meat and poultry, dairy, food diversity, and alcohol consumption $[15,16]$. In the 45 and Up questionnaire, dietary consumption was assessed by short food frequency questions, which have been described in previous research [17, 18]. Each of the questions on diet were previously validated in the Million Women Study [19].

Adequate fruit and vegetable (FV) consumption was identified according to the ADG. Adequate vegetable consumption was identified as $\geq 5.5$ serves per day for males aged $51-70$ years, $\geq 5$ serves per day for males who aged 70 and above; and $\geq 5$ serves per day for females across all age groups. Adequate fruit consumption was identified as $\geq 2$ serves per day for males and females across all age groups. The frequency of food groups for grains, and lean meat and poultry were divided into two groups: lower than mean $(0-5$ times per week for grains, $0-7$ times per week for lean meats and poultry) and higher than mean ( $>5$ times per week for grains, $\geq 7$ times per week for lean meats and poultry). Dairy was categorised as Yes/No. Food diversity was identified if participants consumed all five food groups, i.e., fruit, vegetable, grains, lean meat and poultry, and dairy. Alcohol consumption was identified as Yes/No. Based on ADG, we further generated overall dietary risk scores (Table S1), with range from 0 (the healthiest dietary consumption) to 9 (the unhealthiest dietary consumption).

To evaluate a long-term dietary consumption for each food group and overall dietary consumption, we used both baseline and follow-up dietary data and grouped the participants into four sub-groups (Table S2). In brief, four sub-groups were: a. had healthy diet at baseline and follow-up $(+,+)$, b. had unhealthy diet at baseline and follow-up (-,-), c. had healthy diet at baseline but unhealthy diet at follow-up (+,-), and d. had unhealthy diet at baseline but healthy diet at follow-up $(-,+)$. The detailed explanation for sub-group classifications for each food group and overall dietary risk are shown in Table S2.

\section{Covariates}

We included socio-demographic factors and health behaviour factors as covariates in the analysis. Socio-demographic included age, country of birth, marital status, education and socioeconomic level, and health behaviour factors included smoking and physical activity levels. Country of birth was categorized as Australian versus other countries. Education levels were divided into three categories, i.e., Low: no school certificate or other qualification, and school or intermediate certificate; Medium: high school or leaving certificate; and trade or apprenticeship; and High: certificate or diploma, and university degree or higher. Socioeconomic levels were assessed by Socio-Economic Indexes for Areas (SEIFA), which is based on three quantiles (low, medium, high) of Index of Relative Socio-economic Advantage and Disadvantage [20].

Smoking was identified as never smoke, previous smoker, and current smoker, based on two questions of "Have you ever been a regular smoker?" and "Are you a regular smoker now?". Physical activity was measured using the Active Australia Survey, asking the total time spent on walking, moderate-intensity, and vigorousintensity physical activity in the previous week. Adequate physical activity was identified if people spent $150 \mathrm{~min}$ of moderate intensity physical activity, or $75 \mathrm{~min}$ of vigorous intensity physical activity per week [21].

\section{Statistical analysis}

$\mathrm{N}$ (\%) were used to present the participants' characteristics by three FRAIL categories for males and females. Chi-square tests were used to examine statistical differences between three FRAIL categories and sociodemographic factors for males and females, respectively. The statistical differences between males and females for the prevalence of five FRAIL domains were tested by Chi-square. T-test was used to test mean differences of food group consumption (continuous variables), and Chi-square was used to test differences for categorical food group consumption across baseline and follow-up for males and females, respectively. Multinomial regression models were used to test 1 ) the association between a long-term dietary consumption of each food group and frailty, stratified by sex; and 2) the association between a long-term overall dietary consumption and frailty, stratified by sex. These results are reported in the tables, with Relative Risk Ratios (RRR) and 95\% Confidence Interval (CI) in two models: 1) crude model and 2) adjusted model that after adjustment of socioeconomic factors and health behaviour factors. All analyses were conducted in STATA/SE 14 (StataCorp, USA). 


\section{Results}

\section{Participants characteristics}

There were 15,745 (30.1\%) males and 21,543 (35.5\%) females identified as pre-frail, $1864(3.56 \%)$ males and 2948 (4.86\%) females were identified as frail. Participants characteristics by three FRAIL categories for males and females were shown in Table 1 . Overall, females had a higher percentage of pre-frailty and frailty than males across all socioeconomic factors. Females who aged 80 years and over, widowed, with low education level and lived in low socioeconomic areas had higher percentage of pre-frailty and frailty than male counterparts. Females, who were identifies as per-frail and frail, had higher percentage of CVD, diabetes, and higher overall dietary risks than males. Across five domains of the FRAIL scale, females had a higher percentage of fatigue, resistance, ambulation, and loss of weight than males $(p<$ $0.001)$. No association were found between males and females on illness $(p=0.12)$ (Fig. 1).

\section{The changes of dietary consumption by frailty status and sex}

The changes of each food group consumption across two waves by three FRAIL categories for males and females were shown in Table 2. Overall, we found similar trends in food consumption across three FRAIL

Table 1 Participants characteristics by three FRAlL categories for males and females $(N=113,039)$

\begin{tabular}{|c|c|c|c|c|c|c|c|c|}
\hline & \multicolumn{3}{|l|}{ Males } & \multirow{2}{*}{$\begin{array}{l}p \\
\text { value }\end{array}$} & \multicolumn{3}{|l|}{ Females } & \multirow{2}{*}{$\begin{array}{l}p \\
\text { value }\end{array}$} \\
\hline & Healthy & Pre-frail & Frail & & Healthy & Pre-frail & Frail & \\
\hline Age group & & N (\%) & & & & N (\%) & & \\
\hline 50-64 years & $12,482(74.3)$ & $4026(24.0)$ & $298(1.77)$ & $<0.001$ & $16,179(68.2)$ & $6917(29.2)$ & $628(2.65)$ & $<0.001$ \\
\hline $65-79$ years & $18,514(67.5)$ & $8081(29.5)$ & 847 (3.09) & & $17,527(59.3)$ & $10,795(36.5)$ & $1245(4.21)$ & \\
\hline 80 years + & $3770(46.4)$ & $3638(44.8)$ & 719 (8.85) & & $2467(33.5)$ & $3827(51.9)$ & $1074(14.6)$ & \\
\hline \multicolumn{9}{|l|}{ Country of birth } \\
\hline Australia & $26,397(66.3)$ & $11,959(30.0)$ & $1452(3.65)$ & 0.07 & $28,349(59.5)$ & $16,924(35.5)$ & $2369(4.97)$ & $<0.01$ \\
\hline Other countries & $8187(66.9)$ & $3656(29.9)$ & $394(3.22)$ & & $7664(60.5)$ & $4445(35.1)$ & $553(4.37)$ & \\
\hline \multicolumn{9}{|l|}{ Marital status } \\
\hline Married/partner & $28,778(68.3)$ & $12,043(28.6)$ & $1286(3.05)$ & $<0.001$ & $25,719(64.1)$ & $13,014(32.5)$ & $1367(3.41)$ & $<0.001$ \\
\hline Single/divorce/separated & $3951(61.8)$ & $2141(33.5)$ & $306(4.78)$ & & $5898(56.5)$ & $3912(37.5)$ & $637(6.10)$ & \\
\hline Widowed & $1604(50.1)$ & $1357(42.3)$ & $244(7.61)$ & & $4271(44.5)$ & $4426(46.1)$ & $907(9.44)$ & \\
\hline \multicolumn{9}{|l|}{ Education } \\
\hline Low & $6830(58.6)$ & $4164(35.7)$ & $664(5.70)$ & $<0.001$ & $12,056(53.2)$ & $9049(39.9)$ & $1556(6.87)$ & $<0.001$ \\
\hline Medium & $16,369(66.3)$ & $7404(30.0)$ & $905(3.67)$ & & $13,602(61.0)$ & $7704(34.6)$ & $981(4.40)$ & \\
\hline High & $11,205(72.6)$ & $3976(25.8)$ & $262(1.70)$ & & $10,221(67.6)$ & $4537(30.0)$ & $352(2.33)$ & \\
\hline \multicolumn{9}{|l|}{ SEIFA $^{a}$} \\
\hline Low & $10,119(61.5)$ & $5493(33.4)$ & $856(5.20)$ & $<0.001$ & $11,030(54.3)$ & $7870(38.8)$ & $1411(6.95)$ & $<0.001$ \\
\hline Medium & $10,944(66.7)$ & $4896(29.9)$ & $559(3.41)$ & & $11,383(60.1)$ & $6702(35.4)$ & $843(4.45)$ & \\
\hline High & $11,693(70.8)$ & $4461(27.0)$ & $365(2.21)$ & & $11,731(65.0)$ & $5783(32.0)$ & $545(3.02)$ & \\
\hline \multicolumn{9}{|l|}{ Dietary risk scores } \\
\hline Lower than mean & $11,369(65.4)$ & $5333(30.7)$ & $671(3.86)$ & 0.001 & $16,082(60.1)$ & $9476(35.4)$ & $1192(4.46)$ & $<0.001$ \\
\hline Higher than mean & $23,397(66.8)$ & $10,412(29.8)$ & $1193(3.41)$ & & $20,091(59.2)$ & $12,067(35.6)$ & $1756(5.18)$ & \\
\hline \multicolumn{9}{|l|}{ Alcohol consumption } \\
\hline No & $6849(57.1)$ & $4397(36.7)$ & $749(6.24)$ & $<0.001$ & $12,157(50.9)$ & $9908(41.5)$ & $1828(7.7)$ & $<0.001$ \\
\hline Yes & $27,318(69.4)$ & $10,980(27.9)$ & $1056(2.68)$ & & $23,215(66.1)$ & $10,927(31.1)$ & $971(2.77)$ & \\
\hline \multicolumn{9}{|l|}{ Cardiovascular disease } \\
\hline No & $25,435(71.9)$ & $9236(26.1)$ & $694(1.96)$ & $<0.001$ & $30,458(64.7)$ & $15,267(32.4)$ & $1382(2.93)$ & $<0.001$ \\
\hline Yes & 9331 (54.9) & $6509(38.3)$ & $1170(6.88)$ & & $5715(42.2)$ & $6276(46.3)$ & 1566 (11.6) & \\
\hline \multicolumn{9}{|l|}{ Diabetes } \\
\hline No & $30,806(69.1)$ & $12,533(28.1)$ & $1270(2.85)$ & $<0.001$ & $34,056(61.8)$ & $18,813(34.1)$ & $2250(4.08)$ & $<0.001$ \\
\hline Yes & $3428(48.9)$ & $3003(42.8)$ & $582(8.30)$ & & 1976 (37.2) & 2653 (49.9) & 689 (13.0) & \\
\hline
\end{tabular}




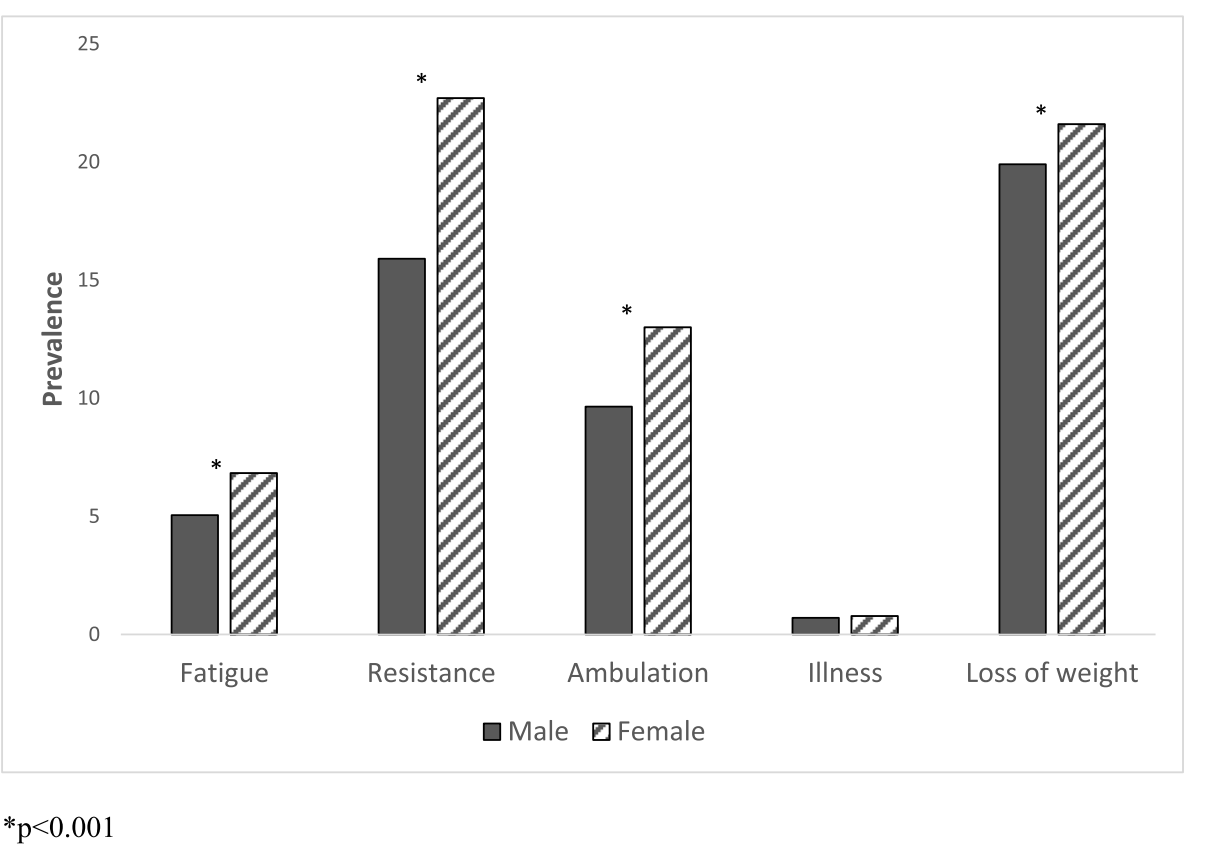

Fig. 1 Prevalence of five domains of the FRAIL scale by sex. ${ }^{*} p<0.001$

categories. This may be due to the overall dietary behaviour changes as peoples' age.

Significant increases in vegetable consumption $(p<$ 0.001 ) were found for males and females who identified as healthy and pre-frail. There were significant increases in fruit consumption for males $(p<0.001)$, and for females who were identified as pre-frail $(p=0.03)$. Significant decreases on grain consumption were found for males and females who identified as healthy $(p<0.001)$, and for female who identified as pre-frail $(p<0.001)$ and frail $(p<0.01)$, however, significant increases were found for males who identified as frail $(p=0.01)$. Significant increases in lean meats and poultry consumption were found for males and females $(p<0.05)$, while significant decreases of alcohol consumption were observed across three FRAIL categories for males and females $(p<$ 0.001). No significant differences were found for dairy and food diversity across two waves for males and females. Males had significant decreases in overall dietary risk scores, while females had significant increases in dietary risk scores across two waves.

\section{The long-term dietary consumption and frailty}

The associations between long-term dietary consumption and frailty are shown in Table 3 . After adjustment of socio-economic and health behaviour factors, no significant associations were found between vegetable consumption and frailty for males. Females with a longterm inadequate vegetable consumption $(R R R=1.34$, $95 \%$ CI: $1.19 ; 1.51$ ), and those with inadequate vegetable consumption at either wave had a higher risk of frailty, compare to females who had a long-term adequate vegetable consumption.

Compared with males and females who had a longterm fruit consumption, males and females with inadequate fruit consumption at both waves or had inadequate fruit consumption at either wave had a higher risk of frailty.

Compared with males and females with a long-term high grain consumption, males who had a long-term low grain consumption (RRR $=1.19,95 \% \mathrm{CI}$ : $1.03 ; 1.37)$ and those who had a low grain consumption at baseline but had high grains consumption at follow-up $(R R R=1.74$, 95\% CI: $1.44 ; 2.09$ ) had a higher risk of frailty. Females who had a long-term low grains consumption ( RRR = 1.30, 95\% CI: $1.16 ; 1.46)$, and those who had a low grains consumption at either wave $(\mathrm{RRR}=1.35,95 \% \mathrm{CI}$ : 1.17 ; $1.57 ; \mathrm{RRR}=1.35,95 \% \mathrm{CI}: 1.15 ; 1.59)$ had a higher risk of frailty.

Compared with males and females with a long-term high lean meats and poultry consumption, males who had a low lean meats and poultry consumption at baseline but had high lean meats and poultry consumption at follow-up had a higher risk of pre-frailty $(R R R=1.07$, 95\% CI:1.01; 1.13) Females who had a high lean meats and poultry consumption at baseline but had low lean meats and poultry consumption at follow-up (RRR = $1.22,95 \%$ CI:1.07; 1.39) had a higher risk of frailty.

Compared with males and females with long-term dairy consumption, males who had dairy consumption at baseline but no dairy consumption at follow-up (RRR = 1.23, 95\% CI: 1.07 ; 1.41) had a higher risk of pre-frailty. 
Table 2 The changes of dietary consumption across baseline and follow-up by three FRAIL categories for males and females

\begin{tabular}{|c|c|c|c|c|c|c|c|c|}
\hline & \multicolumn{4}{|l|}{ Males } & \multicolumn{4}{|c|}{ Females } \\
\hline & & Baseline & Follow-up & $P$ value & & Baseline & Follow-up & $P$ value \\
\hline Vegetable & $N$ & Mean (SD) & Mean (SD) & & $N$ & Mean (SD) & Mean (SD) & \\
\hline Health & 34,766 & $3.45(2.58)$ & $3.59(2.85)$ & $<0.001$ & 36,173 & $4.44(2.60)$ & $4.64(2.91)$ & $<0.001$ \\
\hline Pre-frail & 15,745 & $3.43(2.66)$ & $3.60(2.99)$ & $<0.001$ & 21,543 & $4.41(2.66)$ & $4.54(3.06)$ & $<0.001$ \\
\hline Frail & 1864 & $3.52(2.75)$ & $3.50(3.07)$ & 0.79 & 2948 & $4.18(2.65)$ & $4.13(3.11)$ & 0.45 \\
\hline \multicolumn{9}{|l|}{ Fruit } \\
\hline Health & 33,212 & $1.89(1.43)$ & $1.97(1.56)$ & $<0.001$ & 35,067 & $2.17(1.29)$ & $2.16(1.40)$ & 0.004 \\
\hline Pre-frail & 14,852 & $1.84(1.46)$ & $1.99(1.63)$ & $<0.001$ & 20,749 & $2.11(2.33)$ & $2.15(1.46)$ & 0.03 \\
\hline Frail & 1728 & 1.78 (1.49) & $1.99(1.88)$ & $<0.001$ & 2803 & $2.04(1.38)$ & $2.07(1.61)$ & 0.76 \\
\hline \multicolumn{9}{|l|}{ Grain } \\
\hline Health & 33,080 & $5.05(2.70)$ & $4.96(2.70)$ & $<0.001$ & 34,280 & $4.84(2.66)$ & $4.61(2.73)$ & $<0.001$ \\
\hline Pre-frail & 14,803 & $4.94(2.76)$ & $4.93(2.80)$ & 0.52 & 20,136 & $4.84(2.73)$ & $4.62(2.81)$ & $<0.001$ \\
\hline Frail & 1723 & $4.90(2.85)$ & $5.03(2.75)$ & 0.01 & 2698 & $4.80(2.90)$ & $4.59(2.88)$ & $<0.01$ \\
\hline \multicolumn{9}{|c|}{ Lean meats \& poultry } \\
\hline Health & 34,766 & $6.88(3.96)$ & $7.31(3.53)$ & $<0.001$ & 36,173 & $6.56(3.52)$ & $6.91(3.03)$ & $<0.001$ \\
\hline Pre-frail & 15,745 & $6.80(4.09)$ & 7.19 (3.93) & $<0.001$ & 21,543 & $6.51(3.65)$ & $6.87(3.21)$ & $<0.001$ \\
\hline Frail & 1864 & $6.70(3.98)$ & $7.03(3.79)$ & 0.003 & 2948 & $6.46(3.94)$ & $6.62(3.24)$ & 0.04 \\
\hline \multicolumn{9}{|l|}{ Alcohol } \\
\hline Health & 34,466 & $10.5(11.2)$ & $9.86(10.9)$ & $<0.001$ & 35,707 & $5.13(6.08)$ & $4.89(6.04)$ & $<0.001$ \\
\hline Pre-frail & 15,563 & $10.3(12.4)$ & $8.74(11.4)$ & $<0.001$ & 21,114 & $4.34(6.25)$ & $3.81(5.95)$ & $<0.001$ \\
\hline Frail & 1836 & $10.3(14.1)$ & $7.25(11.8)$ & $<0.001$ & 2883 & $3.25(5.91)$ & $2.43(5.16)$ & $<0.001$ \\
\hline \multicolumn{9}{|c|}{ Dietary risk scores } \\
\hline Health & 34,766 & $4.29(1.48)$ & $4.18(1.48)$ & $<0.001$ & 36,173 & $3.74(1.46)$ & $3,76(1.47)$ & 0.02 \\
\hline Pre-frail & 15,745 & $4.30(1.51)$ & $4.14(1.50)$ & $<0.001$ & 21,543 & $3.75(1.50)$ & $3.77(1.49)$ & 0.04 \\
\hline \multirow[t]{2}{*}{ Frail } & 1864 & $4.29(1.52)$ & $4.10(1.53)$ & $<0.001$ & 2948 & 3.81 (1.53) & $3.92(1.53)$ & $<0.01$ \\
\hline & & Yes & Yes & & & Yes & Yes & \\
\hline Dairy & & N (\%) & N (\%) & & & N (\%) & N (\%) & \\
\hline Health & - & $34,019(66.4)$ & $33,890(66.6)$ & 0.63 & - & $35,392(59.7)$ & $35,411(59.7)$ & 0.89 \\
\hline Pre-frail & - & $15,372(30.0)$ & $15,218(29.9)$ & 0.67 & - & $21,052(35.5)$ & 21,008 (35.4) & 0.80 \\
\hline Frail & - & $1822(3.56)$ & $1802(3.54)$ & 0.88 & - & $2863(4.83)$ & $2882(4.86)$ & 0.79 \\
\hline \multicolumn{9}{|c|}{ Food diversity } \\
\hline Health & - & $25,141(67.8)$ & 25,351 (67.9) & 0.71 & - & $27,346(60.8)$ & $26,884(61.2)$ & 0.30 \\
\hline Pre-frail & - & $10,748(29.0)$ & 10,782 (28.9) & 0.78 & - & $15,603(34.7)$ & 15,132 (34.4) & 0.39 \\
\hline Frail & - & $1189(3.21)$ & $1184(3.17)$ & 0.79 & - & 1998 (4.45) & 1925 (4.38) & 0.64 \\
\hline
\end{tabular}

Females who had dairy consumption at baseline but no dairy consumption at follow-up had a lower risk of frailty (RRR $=0.60,95 \%$ CI: $0.42 ; 0.85$ ), while those had no dairy consumption at baseline but had dairy consumption at follow-up had a higher risk of frailty (RRR = 1.39, 95\% CI:1.04; 1.85).

Males and females who didn't consume a long-term variety of foods, or only had a variety of food at one wave had a higher risk of frailty than those with a longterm variety of food consumption.
Compared with males and females without alcohol consumption, those with a long-term alcohol consumption (males: $R R R=0.49,95 \%$ CI: $0.43 ; 0.55$; females: $\mathrm{RRR}=0.36$; $95 \% \mathrm{CI}: 0.32 ; 0.40)$ and those with no alcohol consumption at baseline but had alcohol consumption at follow-up had a lower risk of frailty (males: $R R R=0.64,95 \%$ CI: 0.46; 0.89; females: $R R R=0.59,95 \%$ CI: 0.47 ; 0.74). However, males with alcohol consumption at baseline but has no alcohol consumption has a higher risk of frailty ( $R R R=1.40,95 \%$ CI: 1.16; 1.69). 
Table 3 The association between a long-term food group consumption and frailty, stratified by sex

\begin{tabular}{|c|c|c|c|c|c|}
\hline \multirow{4}{*}{$\begin{array}{l}\text { Long-term food } \\
\text { grouping }\end{array}$} & \multicolumn{5}{|l|}{ Males } \\
\hline & \multicolumn{5}{|c|}{ Relative Risk Ratio (RRR) } \\
\hline & \multicolumn{3}{|c|}{ Crude model } & \multicolumn{2}{|l|}{ Adjusted model $^{a}$} \\
\hline & Healthy & Pre-frail & Frail & Pre-frail & Frail \\
\hline \multicolumn{6}{|l|}{ Vegetable } \\
\hline,$++(N=4018)$ & 1 & 1 & 1 & 1 & 1 \\
\hline,$--(N=35,302)$ & 1 & $0.86(0.80 ; 0.92)$ & $0.67(0.57 ; 0.79)$ & $0.99(0.91 ; 1.07)$ & $0.96(0.79 ; 1.16)$ \\
\hline,$+-(N=5415)$ & 1 & $0.98(0.90 ; 1.07)$ & $0.94(0.77 ; 1.15)$ & $1.00(0.90 ; 1.10)$ & $1.05(0.83 ; 1.32)$ \\
\hline,$-+(N=7640)$ & 1 & $0.96(0.88 ; 1.04)$ & $0.82(0.68 ; 1.00)$ & $1.03(0.93 ; 1.13)$ & $1.08(0.86 ; 1.35)$ \\
\hline \multicolumn{6}{|l|}{ Fruit } \\
\hline,$++(N=19,046)$ & 1 & 1 & 1 & 1 & 1 \\
\hline,$--(N=14,128)$ & 1 & $1.05(1.00 ; 1.10)$ & $1.31(1.15 ; 1.48)$ & $1.02(0.97 ; 1.08)$ & $1.06(0.92 ; 1.23)$ \\
\hline,$+-(N=6526)$ & 1 & $1.10(1.04 ; 1.18)$ & $1.45(1.24 ; 1.69)$ & $1.06(0.99 ; 1.14)$ & $1.22(1.02 ; 1.45)$ \\
\hline,$-+(N=7395)$ & 1 & $1.21(1.14 ; 1.29)$ & $1.49(1.28 ; 1.72)$ & $1.15(1.07 ; 1.22)$ & $1.29(1.09 ; 1.53)$ \\
\hline \multicolumn{6}{|l|}{ Grain } \\
\hline,$++(N=23,200)$ & 1 & 1 & 1 & 1 & 1 \\
\hline,$--(N=14,730)$ & 1 & $1.00(0.95 ; 1.04)$ & $0.91(0.81 ; 1.02)$ & $1.13(1.07 ; 1.19)$ & $1.19(1.03 ; 1.37)$ \\
\hline,$+-(N=5016)$ & 1 & $1.01(0.95 ; 1.08)$ & $0.86(0.72 ; 1.02)$ & $1.12(1.04 ; 1.21)$ & $1.10(0.90 ; 1.36)$ \\
\hline,$-+(N=4419)$ & 1 & $1.23(1.15 ; 1.32)$ & $1.48(1.26 ; 1.73)$ & $1.32(1.22 ; 1.43)$ & $1.74(1.44 ; 2.09)$ \\
\hline \multicolumn{6}{|c|}{ Lean meats \& poultry } \\
\hline,$++(N=19,663)$ & 1 & 1 & 1 & 1 & 1 \\
\hline,$--(N=13,944)$ & 1 & $1.08(1.03 ; 1.14)$ & $1.16(1.02 ; 1.30)$ & $1.05(1.00 ; 1.11)$ & $1.00(0.87 ; 1.15)$ \\
\hline,$+-(N=8757)$ & 1 & $1.10(1.04 ; 1.16)$ & $1.38(1.21 ; 1.58)$ & $1.04(0.97 ; 1.10)$ & $1.11(0.95 ; 1.30)$ \\
\hline,$-+(N=10,011)$ & 1 & $1.08(1.03 ; 1.14)$ & $1.28(1.12 ; 1.46)$ & $1.07(1.01 ; 1.13)$ & $1.12(0.96 ; 1.30)$ \\
\hline \multicolumn{6}{|l|}{ Dairy } \\
\hline,$++(N=49,950)$ & 1 & 1 & 1 & 1 & 1 \\
\hline,$--(N=202)$ & 1 & $1.18(0.87 ; 1.59)$ & $1.65(0.89 ; 3.06)$ & $1.16(0.83 ; 1.63)$ & $1.88(0.97 ; 3.65)$ \\
\hline,$+-(N=1263)$ & 1 & $1.37(1.22 ; 1.54)$ & $1.28(0.96 ; 1.70)$ & $1.23(1.07 ; 1.41)$ & $0.84(0.59 ; 1.18)$ \\
\hline,$-+(N=960)$ & 1 & $1.10(0.96 ; 1.26)$ & $0.94(0.65 ; 1.35)$ & $1.05(0.89 ; 1.22)$ & $0.89(0.59 ; 1.35)$ \\
\hline \multicolumn{6}{|l|}{ Food diversity } \\
\hline,$++(N=30,718)$ & 1 & 1 & 1 & 1 & 1 \\
\hline,$--(N=4821)$ & 1 & $1.20(1.12 ; 1.28)$ & $1.49(1.27 ; 1.74)$ & $1.22(1.13 ; 1.31)$ & $1.50(1.25 ; 1.80)$ \\
\hline,$+-(N=4127)$ & 1 & $1.21(1.13 ; 1.30)$ & $1.38(1.16 ; 1.64)$ & $1.24(1.14 ; 1.34)$ & $1.27(1.04 ; 1.56)$ \\
\hline,$-+(N=4375)$ & 1 & $1.20(1.12 ; 1.28)$ & $1.42(1.20 ; 1.68)$ & $1.21(1.12 ; 1.31)$ & $1.32(1.08 ; 1.61)$ \\
\hline \multicolumn{6}{|c|}{ Alcohol consumption } \\
\hline,$++(N=8560)$ & 1 & 1 & 1 & 1 & 1 \\
\hline,$--(N=37,426)$ & 1 & $0.68(0.64 ; 0.71)$ & $0.40(0.36 ; 0.45)$ & $0.73(0.69 ; 0.77)$ & $0.49(0.43 ; 0.55)$ \\
\hline,$+-(N=1672)$ & 1 & $0.85(0.76 ; 0.95)$ & $0.58(0.44 ; 0.76)$ & $0.89(0.79 ; 1.02)$ & $0.64(0.46 ; 0.89)$ \\
\hline,$-+(N=3243)$ & 1 & $1.37(1.26 ; 1.49)$ & $1.58(1.35 ; 1.86)$ & $1.28(1.17 ; 1.41)$ & $1.40(1.16 ; 1.69)$ \\
\hline \multicolumn{6}{|l|}{ Females } \\
\hline \multicolumn{6}{|l|}{ Vegetable } \\
\hline,$++(N=15,274)$ & 1 & 1 & 1 & 1 & 1 \\
\hline,$--(N=24,738)$ & 1 & $1.04(0.99 ; 1.08)$ & 1.44 (1.30; 1.59) & $1.03(0.98 ; 1.08)$ & $1.34(1.19 ; 1.51)$ \\
\hline,$+-(N=9780)$ & 1 & $1.08(1.02 ; 1.14)$ & $1.50(1.33 ; 1.69)$ & $1.02(0.96 ; 1.09)$ & $1.27(1.10 ; 1.47)$ \\
\hline,$-+(N=10,872)$ & 1 & $1.01(0.95 ; 1.06)$ & $1.14(1.00 ; 1.29)$ & $1.01(0.96 ; 1.07)$ & $1.10(0.95 ; 1.28)$ \\
\hline
\end{tabular}


Table 3 The association between a long-term food group consumption and frailty, stratified by sex (Continued)

\begin{tabular}{|c|c|c|c|c|c|}
\hline \multirow{4}{*}{$\begin{array}{l}\text { Long-term food } \\
\text { grouping }\end{array}$} & \multicolumn{5}{|l|}{ Males } \\
\hline & \multicolumn{5}{|c|}{ Relative Risk Ratio (RRR) } \\
\hline & \multicolumn{3}{|c|}{ Crude model } & \multicolumn{2}{|l|}{ Adjusted model $^{\mathrm{a}}$} \\
\hline & Healthy & Pre-frail & Frail & Pre-frail & Frail \\
\hline \multicolumn{6}{|l|}{ Fruit } \\
\hline,$++(N=32,170)$ & 1 & 1 & 1 & 1 & 1 \\
\hline,$--(N=10,167)$ & 1 & $1.15(1.09 ; 1.20)$ & $1.47(1.33 ; 1.63)$ & $1.10(1.04 ; 1.16)$ & $1.29(1.14 ; 1.46)$ \\
\hline,$+-(N=7490)$ & 1 & $1.11(1.05 ; 1.17)$ & $1.60(1.43 ; 1.79)$ & $1.10(1.04 ; 1.17)$ & $1.49(1.30 ; 1.70)$ \\
\hline,$-+(N=6627)$ & 1 & $1.16(1.10 ; 1.23)$ & $1.55(1.38 ; 1.75)$ & $1.16(1.09 ; 1.23)$ & $1.60(1.39 ; 1.84)$ \\
\hline \multicolumn{6}{|l|}{ Grain } \\
\hline,$++(N=22,727)$ & 1 & 1 & 1 & 1 & 1 \\
\hline,$--(N=19,095)$ & 1 & $0.93(0.90 ; 0.97)$ & $0.88(0.80 ; 0.96)$ & $1.10(1.05 ; 1.15)$ & $1.30(1.16 ; 1.46)$ \\
\hline,$+-(N=7384)$ & 1 & $1.00(0.95 ; 1.06)$ & $1.00(0.88 ; 1.13)$ & $1.15(1.08 ; 1.22)$ & $1.35(1.17 ; 1.57)$ \\
\hline,$-+(N=5352)$ & 1 & $1.03(0.97 ; 1.10)$ & $1.05(0.91 ; 1.21)$ & $1.13(1.05 ; 1.22)$ & $1.35(1.15 ; 1.59)$ \\
\hline \multicolumn{6}{|c|}{ Lean meats \& poultry } \\
\hline,$++(N=22,408)$ & 1 & 1 & 1 & 1 & 1 \\
\hline,$--(N=17,020)$ & 1 & $1.08(1.03 ; 1.12)$ & $1.22(1.11 ; 1.34)$ & $1.03(0.98 ; 1.08)$ & $1.10(0.98 ; 1.23)$ \\
\hline,$+-(N=10,219)$ & 1 & $1.16(1.10 ; 1.22)$ & $1.39(1.25 ; 1.55)$ & $1.09(1.03 ; 1.26)$ & $1.22(1.07 ; 1.39)$ \\
\hline,$-+(N=11,017)$ & 1 & $1.11(1.06 ; 1.17)$ & $1.19(1.07 ; 1.33)$ & $1.08(1.02 ; 1.14)$ & $1.11(0.97 ; 1.26)$ \\
\hline \multicolumn{6}{|l|}{ Dairy } \\
\hline,$++(N=58,143)$ & 1 & 1 & 1 & 1 & 1 \\
\hline,$--(N=199)$ & 1 & $1.22(0.91 ; 1.63)$ & $1.25(0.67 ; 2.32)$ & $1.19(0.85 ; 1.68)$ & $1.53(0.74 ; 3.15)$ \\
\hline,$+-(N=1164)$ & 1 & $1.18(1.04 ; 1.33)$ & $1.04(0.79 ; 1.38)$ & $0.98(0.85 ; 1.13)$ & $0.60(0.42 ; 0.85)$ \\
\hline,$-+(N=1158)$ & 1 & $1.03(0.91 ; 1.17)$ & $1.36(1.07 ; 1.74)$ & $1.05(0.91 ; 1.21)$ & $1.39(1.04 ; 1.85)$ \\
\hline \multicolumn{6}{|l|}{ Food diversity } \\
\hline,$++(N=36,991)$ & 1 & 1 & 1 & 1 & 1 \\
\hline,$--(N=5380)$ & 1 & $1.15(1.08 ; 1.22)$ & $1.33(1.17 ; 1.52)$ & $1.19(1.11 ; 1.27)$ & $1.42(1.21 ; 1.66)$ \\
\hline,$+-(N=5434)$ & 1 & $1.21(1.14 ; 1.29)$ & $1.57(1.38 ; 1.78)$ & $1.27(1.19 ; 1.36)$ & $1.67(1.44 ; 1.94)$ \\
\hline,$-+(N=4425)$ & 1 & $1.18(1.10 ; 1.26)$ & $1.53(1.33 ; 1.76)$ & $1.20(1.12 ; 1.29)$ & $1.56(1.33 ; 1.84)$ \\
\hline \multicolumn{6}{|c|}{ Alcohol consumption } \\
\hline,$++(N=18,935)$ & 1 & 1 & 1 & 1 & 1 \\
\hline,$--(N=32,160)$ & 1 & $0.56(0.54 ; 0.58)$ & $0.26(0.24 ; 0.28)$ & $0.63(0.60 ; 0.66)$ & $0.36(0.32 ; 0.40)$ \\
\hline,$+-(N=2686)$ & 1 & $0.74(0.68 ; 0.80)$ & $0.51(0.42 ; 0.61)$ & $0.78(0.71 ; 0.86)$ & $0.59(0.47 ; 0.74)$ \\
\hline,$-+(N=4417)$ & 1 & $0.95(0.89 ; 1.12)$ & $0.97(0.85 ; 1.10)$ & $0.97(0.90 ; 1.05)$ & $1.05(0.90 ; 1.22)$ \\
\hline
\end{tabular}

after adjustment of socioeconomic factors and health behaviour factors

The association between a long-term each food group consumption and each domain of the FRAIL scale, stratified by sex are shown in Table S3a \& Table S3b. Overall, the significant associations between food groups and all five domains of FRAIL were observed for males and females, however the associations differed by sex.

The association between overall dietary risk scores and frailty are shown in Table 4 . Females with a long-term unhealthy diet at both waves $(\mathrm{RRR}=1.32,95 \% \mathrm{CI}$ : 1.18 ; 1.49), had unhealthy diet at either wave $(R R R=1.28$, 95\% CI: $1.12 ; 1.47$; RRR $=1.19,95 \%$ CI: $1.04 ; 1.37$ ) had a higher risk of frail than those had a long-term healthy diet. No association were found between total dietary risk and frailty for males.

\section{Discussion}

Our results indicate that the prevalence of frailty was higher in females than in males. People aged 80 and over and people living in rural areas have been oversampled, and the sample also skews to higher socioeconomic status. As age increases, males had better dietary behaviour, while females had worse dietary behaviour, which affected their frailty status. Males and females with a longterm consumption of adequate fruits, high grains or had 
Table 4 The association between overall dietary risk scores and frailty, stratified by sex

\begin{tabular}{|c|c|c|c|c|c|}
\hline \multirow{3}{*}{$\begin{array}{l}\text { Long-term } \\
\text { overall dietary } \\
\text { grouping }\end{array}$} & \multicolumn{5}{|c|}{ Relative Risk Ratio (RRR) } \\
\hline & \multicolumn{3}{|c|}{ Crude model } & \multicolumn{2}{|l|}{ Adjusted model $^{\mathrm{a}}$} \\
\hline & Healthy & Pre-frail & Frail & Pre-frail & Frail \\
\hline \multicolumn{6}{|l|}{ Males } \\
\hline,$++(N=8343)$ & 1 & 1 & 1 & 1 & 1 \\
\hline,$--(N=27,675)$ & 1 & $0.96(0.91 ; 1.01)$ & $0.89(0.78 ; 1.02)$ & $1.02(0.96 ; 1.08)$ & $0.93(0.79 ; 1.08)$ \\
\hline,$+-(N=7327)$ & 1 & $1.01(0.95 ; 1.09)$ & $1.00(0.84 ; 1.18)$ & $1.02(0.95 ; 1.11)$ & $0.92(0.76 ; 1.12)$ \\
\hline,$-+(N=9030)$ & 1 & $1.04(0.98 ; 1.11)$ & $1.11(0.95 ; 1.29)$ & $1.05(0.98 ; 1.13)$ & $1.11(0.93 ; 1.33)$ \\
\hline \multicolumn{6}{|l|}{ Females } \\
\hline,$++(N=16,366)$ & 1 & 1 & 1 & 1 & 1 \\
\hline,$--(N=23,233)$ & 1 & $1.03(0.99 ; 1.08)$ & $1.24(1.12 ; 1.37)$ & $1.06(1.01 ; 1.11)$ & $1.32(1.18 ; 1.49)$ \\
\hline,$+-(N=10,681)$ & 1 & $1.06(1.01 ; 1.12)$ & $1.31(1.17 ; 1.47)$ & $1.04(0.99 ; 1.11)$ & $1.28(1.12 ; 1.47)$ \\
\hline,$-+(N=10,384)$ & 1 & $1.05(1.00 ; 1.11)$ & $1.18(1.05 ; 1.33)$ & $1.04(0.98 ; 1.10)$ & $1.19(1.04 ; 1.37)$ \\
\hline
\end{tabular}

after adjustment of socioeconomic factors and health behaviour factors

a variety of foods were associated with a lower risk of frailty. Females with a long-term consumption of adequate vegetables or had high lean meats and poultry were associated with a lower risk of frailty. In addition, females with a long-term overall healthy diet were associated with a lower risk of frailty. No association were found between overall dietary risk and frailty for males.

Given it has a well-described clinical phenomenon that females live longer than males yet tend to experience greater levels of co-morbidity and disability, these results highlight the importance of sex related frailty. Our results show that the prevalence of frailty was higher in females than in males, which is consistent with previous studies $[19,20]$. For example, a study results from four pooled Australian cohort studies that including 8804 Australian adults aged 65 years and over [22], and a meta-analysis of data from five studies that including 37, 426 participants show that females have higher prevalence of frailty than males [23].

Many studies have shown that males and females have different dietary habits or dietary pattern [24]. For example, the results from Foodborne Diseases Active Surveillance Network Population Survey shows that a higher proportion of men reported eating protein than women, whereas a higher proportion of women consumed fruits and vegetables [25]. These dietary habits may further impact on disease status. Our results support this point, highlighting that with age increases, males had significant decreases in dietary risk scores while females had significant increases in dietary risk scores, in particular those who identified as frail. However, reasons to explain this are not clear. It might be due to that females experienced poor health and living with disease than males as they age [26], which may position them at poor nutrition risk. In addition, we found females who with a long-term lower dietary risk scores had a lower risk of frailty, but there was no association for males. It implies that there is a potential of a stronger link between dietary consumption and frailty in females than males, suggesting sex-specific dietary advice need to be further developed in preventing the frailty.

Although studies have proposed consuming fruit and vegetable (FV) may protect against frailty, the overall evidence is scarce with the study settings and findings heterogeneous [27]. A study including three independent cohorts of community dwelling older adults showed that three portions of fruit per day and two portions of vegetables per day had the strongest associated with the lowest short-term risk of frailty [28]. Studies have proposed four potential mechanisms of the benefit of FV in relation to frailty, including 1) antioxidants in FV play a role in slowing frailty development, 2) phytochemicals contained in FV have strong anti-inflammatory properties that heighten inflammatory stares in older people, 3) FV are rich in nutrients, such as dietary fibre, were strongly linked to a lower incidence of certain diseases (e.g., cardiovascular disease), which is one of the indicators in many frailty measurements, and 4) FV consumption has been associated with stimulating the immune system which is strongly associated with showing frailty development [28]. Some studies assessed the association between specific dietary patterns and frailty $[29,30]$, with FV consumption considered as a sub-analysis, showing that higher FV consumption is associated with a lower incident frailty risk. The sex-specific analysis on the FV and frailty were scarce in the existing literature. Our study showed that the inverse associations between fruits and frailty were observed for both males and females, while the inverse associations between vegetables and frailty were only found for females.

In general, we found dietary behaviours vary as age increases. Many studies have shown that people are likely 
to make different food choices as they get older [31]. This may be mainly due to the physiological changes that associated with age, such as slower gastric emptying, decreased basal metabolic rate, altered taste and smell. Some other factors have been also noted, such as changes on socioeconomic status and diet-related attitudes and beliefs [31]. These changes may increase the risk of diet-related illness.

With age increases, females had a significant reduction in grains consumption (including both whole grain and refined grains). Our results highlighted the benefit of long-term high grains consumption in relation to frailty for males and females. Given the benefit of a long-term grains consumption and frailty, our results suggested that females need to be encouraged to consume more grains in the prevention of frailty. Grains or cereals consumption, often considered as one component in the specific dietary pattern, has been explored in relation to frailty in the existing literature. A recent system review which includes a total of 13 cohort or cross-sectional studies examined the association between dietary patterns and risk of frailty showed that a diet high in fruit, vegetables and whole grains was associated with reduced risk of frailty [32]. However, the association between grain consumption in different diseases may differ by age groups [33], and different types of grains may play different roles in disease prevention for older people [18]. Therefore, dietary advice on specific type of grain consumption by different age groups, for different diseases, needs to be further investigated.

Although dietary protein has always been encouraged to prevent frailty, we found that as age increases, males and females have a significant increase in their lean meats and poultry consumption. Moreover, the benefit of a high lean meats and poultry consumption in relation to frailty was only observed for females. A few studies indicate a protective role of protein supplementation against frailty syndrome, very little evidence regarding the effect of protein supplementation on frailty [1]. A daily $30 \mathrm{~g}$ protein supplement has been suggested to prevent frailty. However, studies have highlighted specific individual characteristics should be considered before prescribing supplements because excess protein can be harmful [1]. Our results suggest that personalized dietary advice, with the person understanding their dietary protein consumption habit, is needed for health professionals in order to provide accurate dietary advice to prevent frailty.

Our results support the previous study that a balanced diet, including consuming a variety of food, is a reasonable approach to prevent frailty. Few studies have indicated that a balance diet could be beneficial in avoiding frailty. For example, adherence to a Mediterranean dietary pattern has been associated with lower odds of frailty
[34]. A systematic review including 19 studies, encompassing a sample of 22,270 older adults suggested the importance of both quantitative (energy intake) and qualitative (nutrient quality) factors of nutrition in the development of frailty.. However, most of these studies were of cross-sectional design [3]. We suggest that more longitudinal studies of understanding the potential role of nutrition in prevention, postponement, or even reversion of frailty syndrome are required [3].

Our results also indicate that males and females had a significant decrease on alcohol consumption as they age. In terms of the relationship between alcohol consumption and frailty, there were inconsistent results across the literature. Some studies indicated the benefits of moderate and harms of no alcohol consumption associated with a higher risk of frailty [35-37], which is in line with our study results. However, a study which followed up the participants across their middle and older ages for 30 years highlighted the relationship between alcohol consumption and frailty transforms during the life course. High alcohol consumption in midlife predicts frailty, whereas the association is reversed in old age [38]. It is possible that alcohol consumption at early age can cause health problems at older age.. Older people with no alcohol consumption may have chosen abstinence due to illness and are therefore susceptible for frailty. Given we only can identify frailty at one time point from our data, further studies using more survey points of follow-up data are required to understand the relationship between long-term alcohol consumption and frailty among the older Australian population.

The strength of the present study is that it involved a large population sample. Tracking a long-term dietary consumption, along with sex-specific analyses provided significant insights on the dietary advice in prevention of frailty for middle-aged and older people. However, there are some limitations. Principally this includes the use of self-reported data, which may have measurement bias on dietary consumption. Second, the measures of dietary risk behaviours are limited, and we were not able to calculate overall caloric intake. A short dietary questionnaire does not capture all relevant food. For example, yogurt should belong to the 'dairy' group, but we were unable to include it in the analysis.. Thirdly, the definition of frailty was identified based on self-reported surveys rather than objective measures. The frailty status can only be identified from the follow-up survey that it is not possible to track the changes of frailty status over time. One of the components in the FRAIL scale is loss of weight. We identified 'loss of weight' if participants' weight decreased by $5 \%$ or more between baseline (2006-2009) and follow-up survey (2012-2015). Given the time interval between baseline and follow-up survey, it is hardly to know the exact time when participants 
losing $5 \%$ or more weight. In addition, depending on age and baseline weight, this time interval may carry different clinical significance in identifying frailty. Further studies of including more survey points of follow-up data will be conducted.

In conclusion, our study suggests that males and females changed their dietary consumption as they age, and these changes affect its association with frailty, particularly for females. The general dietary advice to everyone in prevention of frailty may not be enough. Sexspecific dietary advice, by considering the changes of dietary consumption in males and females as they age, in prevention of frailty needs to be further developed. In addition, understanding individual long-term dietary habit for initial assessment can facilitate health professionals to provide accurate healthy dietary advice to prevent frailty.

\section{Supplementary Information}

The online version contains supplementary material available at https://doi. org/10.1186/s12877-021-02165-2.

Additional file 1: Table S1. Scoring overall dietary scores. Table S2. Group classifications of a long-term dietary consumption. Table S3a. The association between a long-term food group consumption and each domain of the FRAIL scale among males. Table S3b. The association between a long-term dietary group consumption and each domain of the FRAIL scale among females.

\section{Acknowledgements}

This research was completed using data collected through the 45 and Up Study (www.saxinstitute.org.au). The 45 and Up Study is managed by the Sax Institute in collaboration with major partner Cancer Council NSW; and partners: the National Heart Foundation of Australia (NSW Division); NSW Ministry of Health; NSW Government Family \& Community Services Ageing, Carers and the Disability Council NSW; and the Australian Red Cross Blood Service. We thank the many thousands of people participating in the 45 and Up Study.

\section{Authors' contributions}

XX conceptualized the study, conducted the analyses, interpreted the data, and draft the manuscript; SCI and DP contributed to the conceptualisation of the study, reviewed and revised the manuscript.. All authors read and approved the final manuscript.

\section{Funding}

XX is supported by Heart Foundation Post-doctoral Fellowship funded by the Heart Foundation of Australia. SCl is supported by a Heart Foundation Future Leader Fellowship funded by the Heart Foundation of Australia.

\section{Availability of data and materials}

The 45 and Up Study is not publicly available, and it is managed by the Sax Institute. For data access, please contact the 45 and Up Study team at 45 andUp.research@saxinstitute.org.au.

\section{Declarations}

\section{Ethics approval and consent to participate}

The study was in accordance with the Declaration of Helsinki. The conduct of the 45 and Up Study was approved by the University of New South Wales Human Research Ethics Committee. Informed consent was obtained from all participants. Analysis of the 45 and Up Study for the present study was approved by The University of Technology, Sydney (ETH18-2145).
Consent for publication

Not applicable.

\section{Competing interests}

The authors declare that they have no competing interests.

\section{Author details}

${ }^{1}$ School of Population Health, University of New South Wales, Sydney, New South Wales, Australia. ${ }^{2}$ The George Institute for Global Health, Sydney, New South Wales, Australia. ${ }^{3}$ Improving Palliative, Aged and Chronic Care through Clinical Research and Translation Research Centre, Faculty of Health,

University of Technology Sydney, Sydney, New South Wales, Australia.

Received: 20 December 2020 Accepted: 15 March 2021

Published online: 31 March 2021

\section{References}

1. Ma L, Zhang L, Sun F, Li Y, Tang Z. Cognitive function in Prefrail and frail community-dwelling older adults in China. BMC Geriatr. 2019;19(1):53. https://doi.org/10.1186/s12877-019-1056-8.

2. Han B, Li Q, Chen X. Effects of the frailty phenotype on post-operative complications in older surgical patients: a systematic review and meta-analysis. BMC Geriatr. 2019;19(1):141. https://doi.org/10.1186/s12877-019-1153-8.

3. Lorenzo-López L, Maseda A, de Labra C, Regueiro-Folqueira L, RodríguezVillamil JL, Millán-Calenti JC. Nutritional determinants of frailty in older adults: a systematic review. BMC Geriatr. 2017;17(1):108. https://doi.org/10.11 86/s12877-017-0496-2

4. Morley JE, Vellas B, Van Kan GA, Anker SD, Bauer JM, Bernabei R, et al. Frailty consensus: a call to action. J Am Med Dir Assoc. 2013;14(6):392-7. https:// doi.org/10.1016/j.jamda.2013.03.022.

5. Whitelock E, Ensaff $\mathrm{H}$. On your own: older Adults' food choice and dietary habits. Nutrients. 2018;10(4):413. https://doi.org/10.3390/nu10040413.

6. Krok-Schoen JL, Archdeacon Price A, Luo M, Kelly OJ, Taylor CA. Low dietary protein intakes and associated dietary patterns and functional limitations in an aging population: a NHANES analysis. J Nutr Health Aging. 2019;23(4): 338-47. https://doi.org/10.1007/s12603-019-1174-1.

7. Chapman IM. The anorexia of aging. Clin Geriatr Med. 2007;23(4):735-56. https://doi.org/10.1016/j.cger.2007.06.001.

8. Bates C, Prentice A, Finch S. Gender differences in food and nutrient intakes and status indices from the National Diet and nutrition survey of people aged 65 years and over. Eur J Clin Nutr. 1999;53(9):694-9. https://doi.org/1 0.1038/sj.ejcn.1600834.

9. Woodward M. Cardiovascular disease and the female disadvantage. Int J Environ Res Public Health. 2019;16(7):1165. https://doi.org/10.3390/ijerph1 6071165 .

10. Sax Institute. 45 and Up Study 2015. Available from: https://www.sa xinstitute.org.au/our-work/45-up-study/.

11. Banks E, Redman S, Jorm L, Armstrong B, Bauman A, Beard J, et al. Cohort profile: the 45 and up study. Inter J Epidemiol. 2007;37:941-7.

12. Lopez D, Flicker L, Dobson A. Validation of the frail scale in a cohort of older Australian women. J Am Med Dir Assoc. 2012;60:171-3.

13. Gardiner PA, Mishra GD, Dobson A. Validity and responsiveness of the FRAlL scale in a longitudinal cohort study of older Australian women. J Am Med Dir Assoc. 2015;16(9):781-3. https://doi.org/10.1016/j.jamda.2015.05.005.

14. van Kan GA, Rolland YM, Morley JE, Vellas BJ. Frailty: toward a clinical definition. J Am Med Dir Assoc. 2008;9(2):71-2. https://doi.org/10.1016/j.ja mda.2007.11.005.

15. Australian Government. Eat for health: Australian dietary guidelines providing the scientific evidence for healthier Australian diets. Canberra: National Health and Medical Research Council; 2013.

16. Australian Government. Australian guidelines to reduce health risks from drinking alcohol. Canberra: National Health and Medical Research Council; 2020.

17. Mihrshahi S, Ding D, Gale J, Allman-Farinelli M, Banks E, Bauman AE. Vegetarian diet and all-cause mortality: evidence from a large populationbased Australian cohort-the 45 and up study. Prev Med. 2017:97:1-7. https://doi.org/10.1016/j.ypmed.2016.12.044

18. Xu X, Parker D, Inglis SC, Byles J. Can regular long-term breakfast cereals consumption benefits lower cardiovascular diseases and diabetes risk? A longitudinal population-based study. Ann Epidemiol. 2019:37:43-50. https:// doi.org/10.1016/j.annepidem.2019.07.004. 
19. Astell-Burt T, Feng X, Croteau K, Kolt GS. Medicine: influence of neighbourhood ethnic density, diet and physical activity on ethnic differences in weight status: a study of 214,807 adults in Australia. Soc Sci Med. 2013;93:70-7. https://doi.org/10.1016/.socscimed.2013.06.006.

20. Australian Bureau of Statistics is the publisher. The reference is available at: https://www.abs.gov.au/websitedbs/censushome.nsf/home/seifa. Accessed 26 July 2018.

21. Australian Institute of Health and Welfare. The Active Australia Survey: a guide and manual for implementation, analysis and reporting. Cat. no. CVD 22. Canberra: AlHW; 2003.

22. Thompson MQ, Theou O, Karnon J, Adams RJ, Visvanathan R. Frailty prevalence in Australia: findings from four pooled Australian cohort studies. Aust J Ageing. 2018;37(2):155-8. https://doi.org/10.1111/ajag.12483.

23. Gordon E, Peel N, Samanta M, Theou O, Howlett S, Hubbard R. Sex differences in frailty: a systematic review and meta-analysis. Exp Gerontol. 2017;89:30-40. https://doi.org/10.1016/j.exger.2016.12.021.

24. Alkazemi D. Gender differences in weight status, dietary habits, and health attitudes among college students in Kuwait: a cross-sectional study. Nutr Health. 2019;25(2):75-84. https://doi.org/10.1177/0260106018817410.

25. Shiferaw B, Verrill L, Booth H, Zansky SM, Norton DM, Crim S, et al. Sexbased differences in food consumption: foodborne diseases active surveillance network (FoodNet) population survey, 2006-2007. Clin Infect Dis. 2012;54(suppl_5):S453-7. https://doi.org/10.1093/cid/cis247.

26. Australian Institute of Health and Welfare. (2019). The health of Australia's females. Retrieved from https://www.aihw.gov.au/reports/men-women/fema le-health

27. Kojima G, Avgerinou C, lliffe S, Jivraj S, Sekiguchi K, Walters K. Fruit and vegetable consumption and frailty: a systematic review. J Nutr Health Aging. 2018;22(8):1010-7. https://doi.org/10.1007/s12603-018-1069-6.

28. García-Esquinas E, Rahi B, Peres K, Colpo M, Dartigues J-F, Bandinelli S, et al. Consumption of fruit and vegetables and risk of frailty: a dose-response analysis of 3 prospective cohorts of community-dwelling older adults. Am J Clin Nutr. 2016;104(1):132-42. https://doi.org/10.3945/ajcn.115.125781.

29. Rahi B, Ajana S, Tabue-Teguo M, Dartigues J-F, Peres K, Feart C. High adherence to a Mediterranean diet and lower risk of frailty among French older adults community-dwellers: results from the Three-City-Bordeaux study. Clin Nutr. 2018;37(4):1293-8. https://doi.org/10.1016/j.clnu.2017.05.020

30. León-Muñoz LM, Guallar-Castillón P, López-García E, Rodríguez-Artalejo F. Mediterranean diet and risk of frailty in community-dwelling older adults. J Am Med Dir Assoc. 2014;15(12):899-903. https://doi.org/10.1016/j.jamda.2 014.06.013.

31. Drewnowski A, Shultz JM. Impact of aging on eating behaviors, food choices, nutrition, and health status. J Nutr Health Aging. 2001;5:75-9.

32. Rashidi Pour Fard N, Amirabdollahian F, Haghighatdoost F. Dietary patterns and frailty: a systematic review and meta-analysis. Nutr Rev. 2019;77(7):498513. https://doi.org/10.1093/nutrit/nuz007.

33. Xu X, Ling M, Inglis SC, Hickman L, Parker D. Eating and healthy ageing: a longitudinal study on the association between food consumption, memory loss and its comorbidities. Int J Public Health. 2020;65(5):571-82.

34. Bollwein J, Diekmann R, Kaiser MJ, Bauer JM, Uter W, Sieber CC, et al. Dietary quality is related to frailty in community-dwelling older adults. J Gerontol A Biol Sci. 2013;68(4):483-9. https://doi.org/10.1093/gerona/gls204.

35. Seematter-Bagnoud L, Spagnoli J, Büla C, Santos-Eggimann B. Alcohol use and frailty in community-dwelling older persons aged 65 to 70 years. J Frailty Aging. 2014;3(1):9-14. https://doi.org/10.14283/jfa.2014.2.

36. Trevisan C, Veronese N, Maggi S, Baggio G, Toffanello ED, Zambon S, et al. Factors influencing transitions between frailty states in elderly adults: the Progetto Veneto Anziani longitudinal study. J Am Geriatr Soc. 2017;65(1): 179-84. https://doi.org/10.1111/jgs.14515.

37. Ortolá R, García-Esquinas E, León-Muñoz LM, Guallar-Castillón P, ValenciaMartín JL, Galán I, et al. Patterns of alcohol consumption and risk of frailty in community-dwelling older adults. J Gerontol A Biol Sci. 2016;71(2):251-8. https://doi.org/10.1093/gerona/glv125.

38. Strandberg AY, Trygg T, Pitkälä KH, Strandberg TE. Alcohol consumption in midlife and old age and risk of frailty: alcohol paradox in a 30-year followup study. Age Ageing. 2018;47(2):248-54. https://doi.org/10.1093/ageing/a fx165.

\section{Publisher's Note}

Springer Nature remains neutral with regard to jurisdictional claims in published maps and institutional affiliations.

\section{Ready to submit your research? Choose BMC and benefit from}

- fast, convenient online submission

- thorough peer review by experienced researchers in your field

- rapid publication on acceptance

- support for research data, including large and complex data types

- gold Open Access which fosters wider collaboration and increased citations

- maximum visibility for your research: over $100 \mathrm{M}$ website views per year

At BMC, research is always in progress.

Learn more biomedcentral.com/submissions 